\title{
Serum gamma-glutamyltransferase and the overall survival of metastatic pancreatic cancer
}

\author{
Yuanyuan Xiao ${ }^{1,2}$, Haijun Yang ${ }^{3}$, Jian $\mathrm{Lu}^{3}$, Dehui $\mathrm{Li}^{3}$, Chuanzhi Xu ${ }^{1^{*}}$ (D) and Harvey A. Risch ${ }^{2^{*}}$
}

\begin{abstract}
Background: Accumulating evidence suggests that Gamma-glutamyltransferase (GGT) may be involved in cancer occurrence and progression. However, the prognostic role of serum GGT in pancreatic cancer (PC) survival lacks adequate evaluation. In this study, we aimed to analyze the association between serum GGT measured at diagnosis and overall survival (OS) in patients with metastatic PC.

Methods: We identified 320 patients with histopathologically confirmed metastatic pancreatic ductal adenocarcinoma (PDAC) diagnosed during 2015 and 2016 at a specialized cancer hospital in southwestern China. Univariate and multivariate Cox proportional-hazards models were used to determine associations between serum GGT and OS in metastatic PDAC.

Results: Controlled for possible confounding factors, serum GGT was significantly associated with OS: serum GGT > $48 \mathrm{U} / \mathrm{L}$ yielded a hazard ratio of 1.53 (95\% Cl: 1.19-1.97) for mortality risk. A significant dose-response association between serum GGT and OS was also observed. Subgroup analysis showed a possible interaction between GGT and blood glucose level.
\end{abstract}

Conclusion: Serum GGT could be a potential indicator of survival in metastatic PDAC patients. Underlying mechanisms for this association should be investigated.

Keywords: Biomarkers, Pancreatic cancer, Survival

\section{Background}

The enzyme gamma-glutamyltransferase (GGT) plays a key role in glutathione metabolism. Located primarily on the plasma membrane of the cell, it passes amino acids across the cell membrane and transfers glutamyl onto water, peptides and other recipient molecules. Although GGT is nearly omnipresent in human tissues [1], circulating GGT is thought to be derived predominantly from the liver [2]. Therefore, in clinical practice, blood levels are widely used as a sensitive albeit nonspecific diagnostic biomarker for liver dysfunction, hepatitis and excessive alcohol consumption. In the past two decades, elevated GGT levels have also been associated with increased risk

\footnotetext{
* Correspondence: chuanzhixukmu@126.com; harvey.risch@yale.edu ${ }^{1}$ School of Public Health, Kunming Medical University, 1168 West Chunrong Road, Kunming, Yunnan, China

${ }^{2}$ Department of Chronic Disease Epidemiology, Yale School of Public Health, Yale University, New Haven, CT 06520-8034, USA

Full list of author information is available at the end of the article
}

of various chronic diseases, such as cardiovascular events [3], hypertension [4], type II diabetes [5], metabolic syndrome [6], and renal failure [7].

Pro-oxidation and pro-inflammatory properties of GGT have been suggested to underlie etiological mechanisms leading to these adverse disease outcomes [8]. Oxidative stress and inflammation are also pathways implicated in cancer development and progression, and positive GGT associations with risks of various cancer types have been demonstrated by several prospective studies [9-12]. Quite opposite to the abundance of cancer risk studies, relatively few studies have investigated the prognostic role of serum GGT in cancer outcomes: increased serum GGT has been inversely associated with survival in gastric cancer [13], colorectal cancer [14], ovarian cancer [15], breast cancer [16], endometrial cancer $[17,18]$, cervical cancer [19], and renal cell carcinoma [20].

(c) The Author(s). 2019 Open Access This article is distributed under the terms of the Creative Commons Attribution 4.0 International License (http://creativecommons.org/licenses/by/4.0/), which permits unrestricted use, distribution, and reproduction in any medium, provided you give appropriate credit to the original author(s) and the source, provide a link to the Creative Commons license, and indicate if changes were made. The Creative Commons Public Domain Dedication waiver (http://creativecommons.org/publicdomain/zero/1.0/) applies to the data made available in this article, unless otherwise stated. 
Pancreatic cancer (PC) remains one of the most lethal malignant tumors worldwide, with a dismal 5-year survival proportion of less than $5 \%$ [21]. Thus, identification of factors that are of prognostic significance is imperative. In parallel to the aforementioned inverse survival associations with other malignancies, it is possible that serum GGT may also relate to PC survival. Two studies have examined the relations of serum GGT and GGTto-albumin ratio with $\mathrm{PC}$ survival in early stage patients, with positive findings [22, 23]. Few studies have investigated the prognostic relevance of serum GGT in advanced PC patients [24]. Therefore, the aim of this study was to assess the association between serum GGT measured at disease diagnosis and overall survival (OS) in PC patients with metastatic disease.

\section{Methods}

\section{Study design}

After the Institutional Research Ethics Board of Kunming Medical University reviewed and approved the protocol, we performed a retrospective study at The Third Affiliated Hospital of Kunming University, the largest cancer hospital in southwestern China Yunnan province. This hospital has a comprehensive computerized information system that collects, verifies and updates all parts of medical practice-relevant data from inpatients and outpatients on a daily basis, including hospitalization records, imaging examinations, body-fluids tests, drug prescriptions, diagnoses, surgical procedures, etc. In this study, we screened the database for histopathologically confirmed stage IV (metastatic) pancreatic ductal adenocarcinoma (PDAC) patients diagnosed between January 1, 2015 and December 31, 2016. Other information that was obtained from the system included age at diagnosis, sex, blood indicators and chemotherapy data. Information about deaths of the included patients through January 1, 2018 was ascertained by external matching with the Chinese Residents Death Registration System, using the individual personal ID number assigned to every Chinese citizen. Because of the retrospective nature of the study, informed consents from the subjects were waived by the institutional review board.

\section{Variables and definitions}

The outcome of interest in this study was OS. Survival duration was defined as the time between histopathological diagnosis date and death date. Data on baseline serum GGT level for each included patient, measured within 7 days of diagnosis, were extracted from the information system. Considering that nutritional status, liver function, hyperglycemia and systemic inflammation may introduce confounding in analyses of the association between serum GGT and OS, we also extracted baseline test results of several other blood indicators: albumin (ALB), an indicator of nutritional status; total bilirubin
(TBIL) and alanine transaminase (ALT), measurements of liver function; fasting plasma glucose (FPG), a marker of blood glucose level; and neutrophil-to-lymphocyte ratio (NLR), a measure of systemic inflammation. Chemotherapy of the patients was with palliative intention, and was defined as the single or combination use of any of the following commonly used drugs: gemcitabine, nabpaclitaxel, 5-fluorouracil, irinotecan, and oxaliplatin.

\section{Statistical analysis}

Descriptive statistics were used to delineate and compare general characteristics of the study subjects. Univariate and multivariate Cox proportional-hazards models were applied to evaluate the association between baseline serum GGT level and PDAC OS. Subgroup analyses based on chemotherapy, NLR values, and blood glucose levels were performed to examine possible GGT interaction roles. All statistical analyses were done in SAS (version 9.3, SAS Institute Inc., Cary, NC.). Except for the exploratory univariate Cox models, which employed a less strict standard $(p<0.10)$ for initial identification of possible influencing factors, the threshold for nominal statistical significance was defined as a two-tailed probability less than 0.05 .

\section{Results}

\section{General characteristics of PDAC patients}

Over the 2 years of subject eligibility, we identified 357 patients with histologically confirmed metastatic PDAC, of whom 37 had missing values in critical variables. Thus, our analyses included 320 patients. Major characteristics of the patients are described and compared in Table 1. The mean diagnosis age of patients was 65.3 years, males and females comparable. Median survival was 177 days. Although serum GGT can mildly vary by age and sex, in clinical practice, a uniform cut-off of 48 units/liter (U/L) is the most commonly used threshold for defining GGT elevation. We chose this value a priori to dichotomize the PDAC patients based on baseline serum GGT level. We found that, except for age, sex and serum FPG, the various ascertained characteristics were all significantly different between the two groups: compared to patients with normal baseline serum GGT, patients with elevated levels had much shorter median survival (138 versus 281 days), as well as generally higher other blood markers.

\section{Baseline serum GGT and OS of metastatic PDAC}

Product-limit survival curves of elevated and normal baseline serum GGT patients are displayed in Fig. 1. OS of the elevated GGT group was notably inferior to survival of the normal GGT group (log-rank statistic: 23.52, $p=10^{-6}$ ). Univariate Cox proportional-hazards models identified 4 potential prognostic covariates: age at diagnosis, 
Table 1 General characteristics of 320 metastatic PDAC patients

\begin{tabular}{|c|c|c|c|c|}
\hline \multirow[t]{2}{*}{ Characteristics } & All patients $(N=320)$ & $\begin{array}{l}\text { Elevated serum GGT } \\
(\mathrm{GGT}>48 \mathrm{U} / \mathrm{L}, N=183)\end{array}$ & $\begin{array}{l}\text { Normal serum GGT } \\
(\mathrm{GGT} \leq 48 \mathrm{U} / \mathrm{L}, N=137)\end{array}$ & \multirow[t]{2}{*}{$p$ value } \\
\hline & Mean (SD)/Median (SD)/N (\%) & Mean (SD)/Median (SD)/N (\%) & Mean (SD)/Median (SD)/N (\%) & \\
\hline Age at diagnosis (Years) & $65.28(10.05)^{a}$ & $65.93(9.97)^{a}$ & $64.41(10.14)^{a}$ & 0.18 \\
\hline Sex (Male) & $158(49.38)^{c}$ & $93(50.82)^{c}$ & $65(47.45)^{c}$ & 0.57 \\
\hline Palliative chemotherapy (Yes) & $150(46.88)^{c}$ & $63(34.43)^{c}$ & $87(63.50)^{c}$ & $10^{-6.5}$ \\
\hline Survival length (Days) & $177(231.93)^{b}$ & $138(271.65)^{b}$ & $281(234.19)^{b}$ & $10^{-6.0}$ \\
\hline \multicolumn{5}{|l|}{ Baseline serum indicators } \\
\hline ALB (g/L) & $37.00(7.32)^{b}$ & $36.00(6.96)^{b}$ & $39.30(7.12)^{b}$ & $10^{-6.5}$ \\
\hline TBIL $(\mu \mathrm{mol} / \mathrm{L})$ & $15.40(113.14)^{b}$ & $34.80(137.81)^{b}$ & $11.20(15.36)^{b}$ & $10^{-14}$ \\
\hline $\mathrm{ALT}(\mathrm{U} / \mathrm{L})$ & $29.00(107.87)^{b}$ & $58.00(110.61)^{b}$ & $17.10(91.23)^{b}$ & $10^{-8.1}$ \\
\hline $\mathrm{FPG}(\mathrm{mmol} / \mathrm{L})$ & $6.67(3.26)^{b}$ & $6.70(3.78)^{b}$ & $6.42(2.32)^{b}$ & 0.15 \\
\hline NLR (Unit free) & $4.46(8.26)^{b}$ & $5.08(8.13)^{b}$ & $3.74(8.45)^{b}$ & $10^{-2.3}$ \\
\hline GGT $(U / L)$ & $85.5(393.02)^{b}$ & NA & NA & NA \\
\hline
\end{tabular}

${ }^{a}$ Mean with standard deviation (SD)

b Median with standard deviation (SD)

${ }^{\mathrm{c}}$ Frequency with proportion (\%)

palliative chemotherapy, baseline FPG and baseline GGT. With multivariate adjustment, only age at diagnosis, FPG and GGT remained significant. Age at diagnosis was positively associated with mortality: the adjusted hazard ratio (HR) was 1.08 (95\% CI 1.01-1.15) per 5 years increase; elevated baseline FPG and serum GGT were associated with 1.39- (95\% CI: $1.08-1.79)$ and 1.53- (95\% CI: $1.19-1.97)$ fold mortality, respectively (Table 2).

We divided PDAC patients into 4 strata by quartile of baseline serum GGT: $\mathrm{Q}_{1}(\mathrm{GGT}<30.0 \mathrm{U} / \mathrm{L}), \mathrm{Q}_{2}(30.0 \mathrm{U} /$ $\mathrm{L} \leq \mathrm{GGT}<85.5 \mathrm{U} / \mathrm{L}), \quad \mathrm{Q}_{3} \quad(85.5 \mathrm{U} / \mathrm{L} \leq \mathrm{GGT}<338.0 \mathrm{U} / \mathrm{L})$, and $\mathrm{Q}_{4}(\mathrm{GGT} \geq 338.0 \mathrm{U} / \mathrm{L})$. By using $\mathrm{Q}_{1}$ as the reference group, controlling for age at diagnosis, palliative chemotherapy, baseline FPG and baseline ALB, we found that the adjusted $\mathrm{HRs}$ for $\mathrm{Q}_{2}$ through $\mathrm{Q}_{4}$ were $1.36(95 \% \mathrm{CI}$ : 0.96-1.93), 1.53 (95\% CI: $1.07-2.19$ ), and 1.76 (95\% CI: 1.24-2.49), respectively. The multiplicative continuous dose-response association between GGT and OS was statistically significant: every 10-fold increase in GGT was associated with a HR of 1.33 (95\% CI: 1.09-1.61), and the $p$ value for this continuous trend was 0.0043 (Fig. 2).

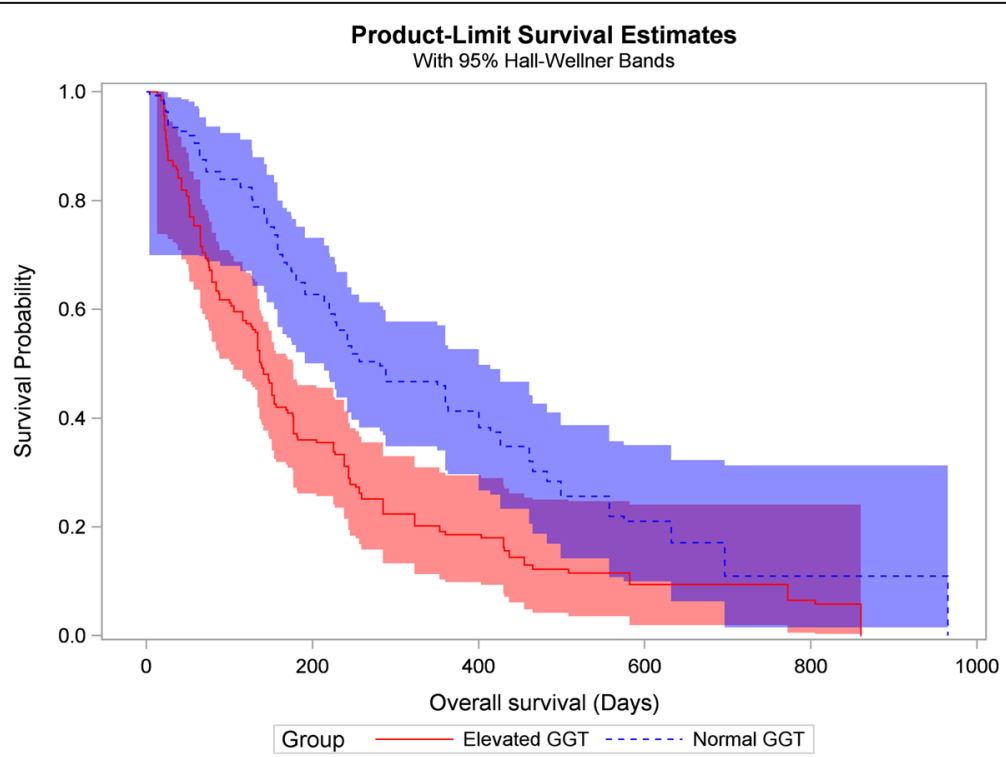

Fig. 1 Kaplan-Meier survival curves for metastatic PDAC patients with elevated and normal baseline serum GGT levels. Log-rank statistic $x^{2}=23.5$, $p=10^{-6}$. PDAC, pancreatic ductal adenocarcinoma; GGT, Gamma-glutamyltransferase 
Table 2 Univariate and multivariate Cox proportional hazards model results

\begin{tabular}{|c|c|c|c|c|}
\hline \multirow[t]{2}{*}{ Covariates } & \multicolumn{2}{|l|}{ Univariate Cox model } & \multicolumn{2}{|l|}{ Multivariate Cox model } \\
\hline & Crude HR (95\% Cl) & $p$ value & Adjusted HR (95\% Cl) & $p$ value \\
\hline Age at diagnosis ( +5 years) & $1.10(1.03,1.16)$ & $10^{-2.9}$ & $1.08(1.01,1.15)$ & $10^{-1.7}$ \\
\hline Sex (Male) & $1.02(0.81,1.29)$ & 0.87 & & \\
\hline Palliative chemotherapy (Yes) & $0.69(0.54,0.87)$ & $10^{-2.9}$ & $0.81(0.62,1.04)$ & 0.10 \\
\hline Baseline serum ALB (< $35 \mathrm{~g} / \mathrm{L})$ & $1.25(0.98,1.59)$ & 0.10 & & \\
\hline Baseline serum TBIL (> $20.5 \mu \mathrm{mol} / \mathrm{L})$ & $1.19(0.93,1.50)$ & 0.16 & & \\
\hline Baseline serum ALT (> $60 \mathrm{U} / \mathrm{L}$ ) & $1.17(0.91,1.50)$ & 0.22 & & \\
\hline Baseline serum NLR (+ 10) & $1.10(0.97,1.25)$ & 0.15 & & \\
\hline Baseline FPG ( $\geq 7.0 \mathrm{mmol} / \mathrm{L})$ & $1.32(1.04,1.68)$ & $10^{-1.7}$ & $1.39(1.08,1.79)$ & $10^{-2.0}$ \\
\hline Baseline serum GGT (> 48 U/L) & $1.78(1.40,2.26)$ & $10^{-6.0}$ & $1.53(1.19,1.97)$ & $10^{-3.2}$ \\
\hline
\end{tabular}

\section{Subgroup analysis}

We further performed a small series of subgroup analyses based on GGT stratification by categories of palliative chemotherapy, baseline FPG and NLR. No obvious interaction was found between palliative chemotherapy, baseline NLR and serum GGT. However, an appreciable difference in the GGT-OS association was found when metastatic PDAC patients were dichotomized by baseline FPG: in patients with elevated baseline FPG (defined as $\geq 7.0 \mathrm{mmol} / \mathrm{L}$ ), GGT was not associated with OS, but in patients with normal baseline FPG (defined as $<7.0$ $\mathrm{mmol} / \mathrm{L}$ ), elevated serum GGT was associated with 2.14fold mortality (95\% CI: 1.48-3.09) (Table 3). This interaction did not reach statistical significance however $(p=$ 0.07).

Among the 320 PDAC patients, 76 and 97 were additionally measured for baseline $\mathrm{C}$-reactive protein (CRP)

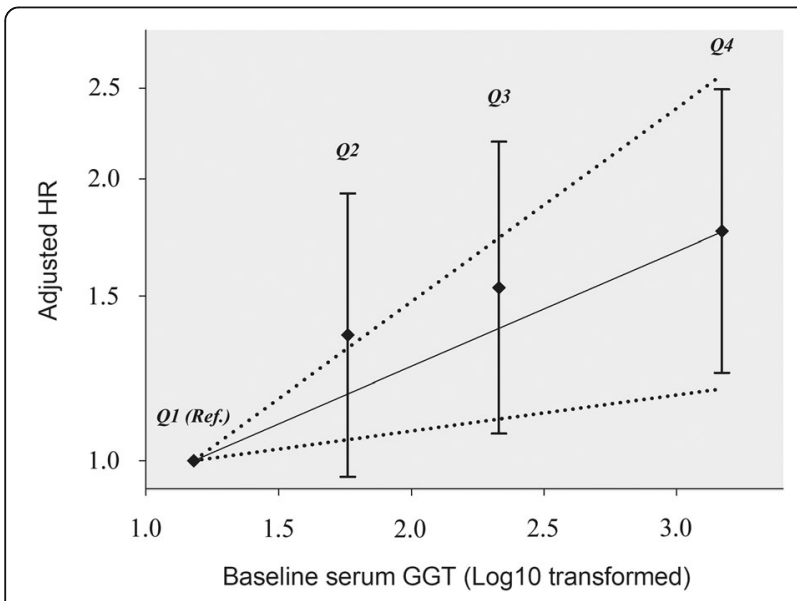

Fig. 2 Dose-response association between baseline serum GGT and the OS in metastatic PDAC patients. Adjusted for age at diagnosis, palliative chemotherapy, baseline ALB, and baseline FPG. The estimated dose-response trend and its 95\% confidence band are given in solid and dotted lines, respectively. The Y-axis represents $\log _{10}(\mathrm{HR})$, thus intervals are not equally spaced. GGT, Gammaglutamyltransferase; ALB, albumin; FPG, fasting plasma glucose and carbohydrate antigen 19-9 (CA19-9), respectively. Correlation analysis revealed that serum GGT was positively associated with CA19-9 $\left(r=0.43, \quad p=10^{-3.9}\right)$, whereas the relationship between GGT and CRP was negligible $(r=-0.01, p=0.97)$. Considering the appreciable correlation between GGT and CA19-9, we fitted a multivariate Cox regression model that included both CA19-9 and GGT in the subset of the 97 PDAC patients with baseline CA19-9 measurements: elevated GGT was still a significant prognostic factor $(\mathrm{HR}=1.53$, 95\% CI: 1.10-2.13), and CA19-9 was not significantly associated with OS (HR $=1.50,95 \%$ CI: 0.78-2.85).

\section{Discussion}

In this study, we examined the prognostic relevance of serum GGT measured at diagnosis among histopathologically diagnosed metastatic PDAC patients. We found that baseline serum GGT was positively associated with OS: compared to patients with normal GGT, elevated GGT showed a $54 \%$ increase in mortality hazard. A previous study by Engelken et al. also reported a significant association between elevated GGT and deteriorated survival in unresectable PDAC patients. However, those authors adopted an unusual 3.4-fold higher cut-off

Table 3 Subgroup analysis results by chemotherapy, baseline FPG and NLR

\begin{tabular}{|c|c|c|c|}
\hline \multirow[t]{2}{*}{ Stratification variable } & \multirow[t]{2}{*}{ Stratum } & \multicolumn{2}{|c|}{ Elevated serum GGT (> $48 \mathrm{U} / \mathrm{L})$} \\
\hline & & $\begin{array}{l}\text { Adjusted HR } \\
(95 \% \mathrm{Cl})\end{array}$ & $\begin{array}{l}\text { Interaction } \\
p \text {-value }\end{array}$ \\
\hline \multirow[t]{2}{*}{ Palliative chemotherapy } & Yes & $1.70(1.16,2.51)^{a}$ & 0.84 \\
\hline & No & $1.61(1.10,2.34)^{a}$ & \\
\hline \multirow[t]{2}{*}{ Baseline NLR } & $\geq 4.46$ & $1.38(0.95,2.00)^{b}$ & 0.91 \\
\hline & $<4.46$ & $1.42(0.99,2.03)^{b}$ & \\
\hline \multirow[t]{2}{*}{ Baseline FPG } & $\geq 7.0 \mathrm{mmol} / \mathrm{L}$ & $1.31(0.90,1.92)^{c}$ & 0.07 \\
\hline & $<7.0 \mathrm{mmol} / \mathrm{L}$ & $2.14(1.48,3.09)^{c}$ & \\
\hline
\end{tabular}

adjusted for age at diagnosis, baseline NLR, baseline FPG

${ }^{\mathrm{b}}$ Adjusted for age at diagnosis, palliative chemotherapy, baseline FPG

c Adjusted for age at diagnosis, palliative chemotherapy, baseline NLR 
(165 U/L) to dichotomize GGT level [24]. Our findings were further supported by an appreciable doseresponse trend of increasing mortality hazard with increasing GGT level. This association is of potential clinical significance: as a prognostic factor. A possible role of serum GGT in cancer survival should be investigated, in the hope of elucidating modifiable survival mechanisms in these cancer patients.

Laboratory studies the involvement of serum GGT in metastatic PDAC prognosis. First, evidence supports a role of inflammation in cancer progression. For example, nuclear factor $-\mathrm{kB}(\mathrm{NF}-\mathrm{kB})$ is involved in inflammationinduced tumor growth [25]. The association of GGT with inflammation has been observed bidirectionally: elevated serum GGT can reflect inflammation-related oxidative stress [26], or can be a consequence of inflammatory cytokines such as tumor necrosis factor alpha [20]. However, our observed mortality association with serum GGT was not affected by adjustment for baseline NLR, a sensitive marker of systemic inflammation, raising the possibility that mechanisms other than inflammation may also exist in this association. For example, GGT may directly participate in the progression of cancer, as studies on melanoma cells found that elevated GGT activity resulted in a growth advantage both in vitro and in vivo [27]. Moreover, a newly published study using gene-set enrichment analysis (GSEA) reported that in gastric cancer patients, GGT was significantly associated with EMT, KRAS, SRC and PKCA signaling pathways [13], which are involved in cancer progression and metastasis [28, 29].

The connection between hyperglycemia and cancer progression has been well established [30]. Our study results are consistent with this association, as we observed that elevated baseline fasting glucose level was associated with increased mortality hazard. However, subgroup analysis in strata of FPG suggested that serum GGT was significantly associated with increased mortality hazard primarily in patients with normal blood glucose levels rather than in their hyperglycemic counterparts. A reasonable hypothesis could be that normal rather than increased plasma glucose level may enhance the bioactivity of serum GGT. However, we cannot find direct laboratory evidence to support this mechanism. Two previous studies have examined the association between serum GGT and glucose levels: one reported a positive association [31], whereas the other reached a nonsignificant conclusion [32]. Another possible explanation could be a pro-inflammatory effect of hypoglycemia that has been noted recently [33,34], as induced inflammation may exacerbate of the association with elevated GGT. Nevertheless, it is also understood that hyperglycemia has a similar pro-inflammation propensity $[35,36]$. Therefore, this issue needs to be further explored.
If validated, the findings of this study may have clinical significance. As an identified prognostic factor, serum GGT level in metastatic PDAC patients could be periodically monitored. As an accompanying indicator of disease progression, serum GGT could help to predict more imminent mortality. On the other hand, if serum GGT independently promotes disease progression, then reducing its level could be a potential strategy in improving survival. Although currently only toxic GGT inhibitors are available $[37,38]$, low toxicity analogs that can be used in humans are in the process of development.

The strength of our study is the comparatively large sample size for this uncommon disease. Nevertheless, several limitations should also be noted. First, all of our study subjects were metastatic PDAC patients chosen from a single Chinese institution, thus generalization of the study results to PC patients with other disease stages or of other ethnic backgrounds should be made cautiously. Second, although we included some potentially important factors for adjustment of the study results, other potential confounders for which we had no information, such as tumor location, smoking history, and alcohol consumption, were not adjusted. Therefore, some unadjusted confounding biases could still exist.

\section{Conclusions}

Increased baseline GGT was associated with lower OS of metastatic PDAC patients. Some evidence for mortality interaction was observed between blood glucose level and serum GGT. Our study results suggest that serum GGT might be used as an indicator to identify late stage PDAC patients with increased mortality hazard. These findings warrant corroboration by studies with larger sample sizes, and mechanisms to explain this association also need to be investigated.

\section{Abbreviations}

ALB: Albumin; ALT: Alanine transaminase; FPG: Fasting plasma glucose; GGT: Gamma-glutamyltransferase (GGT); HR: Hazard ratio; NLR: Neutrophil-tolymphocyte ratio; PDAC: Pancreatic adenocarcinoma; TBIL: Total bilirubin

\section{Acknowledgments \\ None.}

\section{Authors' contributions}

YX, CX, and HAR conceptualized the study. HY, JL and DL collected and sorted the data. $Y X, J L$, and DL performed data analysis. $Y X$ drafted the manuscript, HY, JL, DL, CX, and HAR critically revised the paper. All authors read and approved the final manuscript.

\section{Funding}

This study was supported by National Natural Science Foundation of China (No. 81703324), Yunnan Applied Basic Research Projects-Kunming Medical University Union Foundation (Grant No. 2018FE001(-132)), and the China Scholarship Council (Grant No. 201808535098). The funding organizations had no role on the design and conduct of the study; collection, management, analysis, and interpretation of the data; preparation, review, or approval of the manuscript; and decision to submit the manuscript for publication. 


\section{Availability of data and materials}

The datasets analyzed in the current study are not publicly available due to confidentiality agreements, but are available from the corresponding author subject to approval by the Institutional Research Ethics Board of Kunming University.

\section{Ethics approval and consent to participate}

This study was approved by Institutional Research Ethics Board of Kunming University. Because of its retrospective nature, as well as that no individually identifiable or sensitive information was involved, informed consent from all patients was waived

\section{Consent for publication}

Not applicable.

\section{Competing interests}

The authors declare that they have no competing interests.

\section{Author details}

'School of Public Health, Kunming Medical University, 1168 West Chunrong Road, Kunming, Yunnan, China. ${ }^{2}$ Department of Chronic Disease Epidemiology, Yale School of Public Health, Yale University, New Haven, CT 06520-8034, USA. ${ }^{3}$ The Third Affiliated Hospital of Kunming Medical University, Kunming, Yunnan, China.

Received: 22 May 2019 Accepted: 10 October 2019 Published online: 29 October 2019

\section{References}

1. Goldberg DM. Structural, functional, and clinical aspects of gammaglutamyltransferase. CRC Crit Rev Clin Lab Sci. 1980;12:1-58.

2. Whitfield JB. Gamma-glutamyltransferase. Crit Rev Clin Lab Sci. 2001;38:263355.

3. Emdin M, Passino C, Pompella A, Paolicchi A. Gammaglutamyltransferase as a cardiovascular risk factor. Eur Heart J. 2006;27:2145-6.

4. Lee DH, Jacobs DR Jr, Gross M, Kiefe Cl, Roseman J, Lewis CE, et al. Gammaglutamyltransferase is a predictor of incident diabetes and hypertension: the coronary artery risk development in young adults (CARDIA) study. Clin Chem. 2003:49:1358-66.

5. Lee DH, Silventoinen K, Jacobs DR Jr, Jousilahti P, Tuomileto J. Gammaglutamyltransferase, obesity, and the risk of type 2 diabetes: observational cohort study among 20,158 middle-aged men and women. J Clin Endocrinol Metab. 2004;89:5410-4.

6. Lee DS, Evans JC, Robins SJ, Wilson PW, Albano I, Fox CS, et al. Gamma glutamyl transferase and metabolic syndrome, cardiovascular disease, and mortality risk: the Framingham heart study. Arterioscler Thromb Vasc Biol. 2007:27:127-33.

7. Ryu S, Chang Y, Kim DI, Kim WS, Suh BS. Gammaglutamyltransferase as a predictor of chronic kidney disease in nonhypertensive and nondiabetic Korean men. Clin Chem. 2007:53:71-7.

8. Emdin M, Pompella A, Paolicchi A. Gamma-glutamyltransferase, atherosclerosis, and cardiovascular disease: triggering oxidative stress within the plaque. Circulation. 2005;112:2078-80.

9. Mok Y, Son DK, Yun YD, Jee SH, Samet JM. Y-Glutamyltransferase and cancer risk: the Korean cancer prevention study. Int J Cancer. 2016:138:311-9.

10. Kunutsor SK, Laukkanen JA. Gamma-glutamyltransferase and risk of prostate cancer: findings from the KIHD prospective cohort study. Int J Cancer. 2017; 140:818-24.

11. Strasak AM, Goebel G, Concin H, Pfeiffer RM, Brant $L$, Nagel G, et al. Prospective study of the association of serum gamma-glutamyltransferase with cervical intraepithelial neoplasia III and invasive cervical cancer. Cancer Res. 2010:70:3586-93.

12. Van Hemelrijck M, Jassem W, Walldius G, Fentiman IS, Hammar N, Lambe M, et al. Gamma-glutamyltransferase and risk of cancer in a cohort of 545,460 persons-the Swedish AMORIS study. Eur J Cancer. 2011;47:2033-41.

13. Wang Q, Shu X, Dong Y, Zhou J, Tang R, Shen J, et al. Tumor and serum gamma-glutamyl transpeptidase, new prognostic and molecular interpretation of an old biomarker in gastric cancer. Oncotarget. 2017:8: 36171-84.
14. He WZ, Guo GF, Yin CX, Jiang C, Wang F, Qiu HJ, et al. Gamma-glutamy transpeptidase level is a novel adverse prognostic indicator in human metastatic colorectal cancer. Color Dis. 2013;15:e443-52.

15. Grimm C, Hofstetter G, Aust S, Mutz-Dehbalaie I, Bruch M, Heinze G, et al. Association of gamma-glutamyltransferase with severity of disease at diagnosis and prognosis of ovarian cancer. Br J Cancer. 2013;109: 610-4.

16. Staudigl C, Concin N, Grimm C, Pfeiler G, Nehoda R, Singer CF, et al. Prognostic relevance of pretherapeutic gamma-glutamyltransferase in patients with primary metastatic breast cancer. PLoS One. 2015;10: e0125317.

17. Edlinger M, Concin N, Concin H, Nagel G, Ulmer H, Göbel G. Lifestyle-related biomarkers and endometrial cancer survival: elevated gammaglutamyltransferase as an important risk factor. Cancer Epidemiol. 2013;37: 156-61.

18. Seebacher V, Polterauer S, Grimm C, Rahhal J, Hofstetter G, Bauer EM, et al. Prognostic significance of gamma-glutamyltransferase in patients with endometrial cancer: a multi-Centre trial. Br J Cancer. 2012;106:1551-5.

19. Polterauer S, Hofstetter G, Grimm C, Rahhal J, Mailath-Pokorny M, Kohl M, et al. Relevance of gamma-glutamyltransferase-a marker for apoptotic balance-in predicting tumor stage and prognosis in cervical cancer. Gynecol Oncol. 2011;122:590-4.

20. Luo C, Xu B, Fan Y, Yu W, Zhang Q, Jin J. Preoperative gammaglutamyltransferase is associated with cancer-specific survival and recurrence-free survival of nonmetastatic renal cell carcinoma with venous tumor thrombus. Biomed Res Int. 2017;2017:3142926.

21. Ryan DP, Hong TS, Bardeesy N. Pancreatic adenocarcinoma. N Engl J Med. 2014:371:2140-1.

22. Zhou B, Zhan C, Wu J, Liu J, Zhou J, Zheng S. Prognostic significance of preoperative gamma-glutamyltransferase to lymphocyte ratio index in nonfunctional pancreatic neuroendocrine tumors after curative resection. Sci Rep. 2017:7:13372

23. Li S, Xu H, Wu C, Wang W, Jin W, Gao H, et al. Prognostic value of $\gamma^{-}$ glutamyltransferase-to-albumin ratio in patients with pancreatic ductal adenocarcinoma following radical surgery. Cancer Med. 2019;8:572-84.

24. Engelken FJ, Bettschart V, Rahman MQ, Parks RW, Garden OJ. Prognostic factors in the palliation of pancreatic cancer. Eur J Surg Oncol. 2003;29:368-73.

25. Karin M, Greten FR. NF-kappaB: linking inflammation and immunity to cancer development and progression. Nat Rev Immunol. 2005;5:749-59.

26. Corti A, Franzini M, Paolicchi A, Pompella A. Gamma-glutamyltransferase of cancer cells at the crossroads of tumor progression, drug resistance and drug targeting. Anticancer Res. 2010;30:1169-81.

27. Franzini M, Corti A, Lorenzini E, Paolicchi A, Pompella A, De Cesare M, et al. Modulation of cell growth and cisplatin sensitivity by membrane gammaglutamyltransferase in melanoma cells. Eur J Cancer. 2006;42:2623-30

28. Lewis AD, Hayes JD, Wolf CR. Glutathione and glutathionedependent enzymes in ovarian adenocarcinoma cell lines derived from a patient before and after the onset of drug resistance: intrinsic differences and cell cycle effects. Carcinogenesis. 1988;9:1283-7.

29. Hanigan MH, Gallagher BC, Townsend DM, Gabarra V. Gammaglutamyl transpeptidase accelerates tumor growth and increases the resistance of tumors to cisplatin in vivo. Carcinogenesis. 1999;20:553-9.

30. Ryu TY, Park J, Scherer PE. Hyperglycemia as a risk factor for cancer progression. Diabetes Metab J. 2014;38:330-6.

31. Whitfield JB. Gamma glutamyl transferase. Crit Rev Clin Lab Sci. 2001:38: 263-355.

32. Franzini $M$, Fornaciari I, Rong J, Larson MG, Passino C, Emdin $M$, et al. Correlates and reference limits of plasma gammaglutamyltransferase fractions from the Framingham heart study. Clin Chim Acta. 2013;417: $19-25$.

33. Dandona P, Chaudhuri A, Dhindsa S. Proinflammatory and prothrombotic effects of hypoglycemia. Diabetes Care. 2010;33:1686-7.

34. Ratter JM, Rooijackers HM, Tack CJ, Hijimans AGM, Netea MG, de Galan BE, et al. Proinflammatory effects of hypoglycemia in humans with or without diabetes. Diabetes. 2017;66:1052-61.

35. Aljada A, Ghanim H, Mohanty P, Syed T, Bandyopadhyay A, Dandona P. Glucose intake induces an increase in activator protein 1 and early growth response 1 binding activities, in the expression of tissue factor and matrix metalloproteinase in mononuclear cells, and in plasma tissue factor and matrix metalloproteinase concentrations. Am J Clin Nutr. 2004;80:51-7 
36. Ceriello A. Postprandial hyperglycemia and diabetes complications: is it time to treat? Diabetes. 2005;54:1-7.

37. Han L, Hiratake J, Kamiyama A, Sakata K. Design, synthesis, and evaluation of gamma-phosphono diester analogues of glutamate as highly potent inhibitors and active site probes of gamma-glutamyl transpeptidase. Biochemistry. 2007:46:1432-7.

38. Mena S, Benlloch M, Ortega A, Carretero J, Obrador E, Asensi M, et al. BCl-2 and glutathione depletion sensitizes B16 melanoma to combination therapy and eliminates metastatic disease. Clin Cancer Res. 2007;13:2658-66.

\section{Publisher's Note}

Springer Nature remains neutral with regard to jurisdictional claims in published maps and institutional affiliations.

Ready to submit your research? Choose BMC and benefit from:

- fast, convenient online submission

- thorough peer review by experienced researchers in your field

- rapid publication on acceptance

- support for research data, including large and complex data types

- gold Open Access which fosters wider collaboration and increased citations

- maximum visibility for your research: over $100 \mathrm{M}$ website views per year

At $B M C$, research is always in progress.

Learn more biomedcentral.com/submissions 